\title{
Topological charges and the genus of surfaces
}

\author{
Luis J. Boya * \\ Antonio J. Seguí-Santonja ${ }^{\dagger}$ \\ Departamento de Física Teórica. Facultad de Ciencias. \\ Universidad de Zaragoza. 50009-Zaragoza, Spain
}

November 29, 2017

\begin{abstract}
We show that the topological charge of the $n$-soliton solution of the sine-Gordon equation $n[\phi]=\left[\int \partial_{x} \phi\right] / 2 \pi$ is related to the genus $g>1$ of a constant negative curvature compact surface described by this configuration. The relation is $n=2(g-1)$, where $n=2 \nu$ is even. The moduli space of complex dimension $B_{g}=3(g-1)$ corresponds precisely to the freedom to choosing the configuration with $n$ solitons of arbitrary positions and velocities. We speculate also that the odd soliton states will describe the unoriented surfaces.
\end{abstract}

Keywords: Solitons; Topological charges; (Riemann) Surfaces; Moduli spaces. 1991 MSC :30F, 32G 15, 35Q 53

PACS : $02.40 \mathrm{Hw}$ and $03.50 \mathrm{Kk}$.

\section{Introduction}

The sine-Gordon equation [1], [2]

$$
\phi_{t t}-\phi_{x x}=-\sin \phi
$$

enjoys a great importance in physics:

1. In the lagrangian formalism it presents spontaneous breaking of the discrete $Z$ symmetry $\phi \rightarrow \phi+2 \pi k$ and exhibits the attendant soliton and

*e-mail address: luisjo@posta.unizar.es

$\dagger$ e-mail address:segui@posta.unizar.es 
multisoliton phenomenon. The single static soliton is given as solution of the first order (Bogomolny) equation; if $V(\phi)=W^{2}(\phi) / 2$,

$$
\phi^{\prime} \equiv W(\phi)=\sin \phi
$$

has as solution the profile

$$
\phi(x)=4 \arctan \exp \left(x-x_{0}\right) .
$$

2. The sine-Gordon equation has an auto-Bäcklund transformation [3] which makes it possible to obtain the general $n$-soliton solution [1]. The theory is exactly soluble also by the Inverse Spectral Transform method [5]. The general solution contains multiple soliton/antisoliton configurations, as well as breathers (soliton-antisoliton bound states) and background noise. The quantization is factible [6], [7] and indeed the formulas of the WKB approximation are already exact [8].

3. The quantized sine-Gordon theory is equivalent to the massive Thirring model [9] ; in fact, this duality, already conjectured by Skyrme [10], is the first case found of bosonization of field theories with fermions. Explicit soliton operators in terms of the $\phi$ field were also first exhibited here by Mandelstam [11, an example of the later much-studied vertex operators. The quantum theory can also be made supersymmetric [12].

4. The discrete $Z$ symmetry mentioned above can be seen as a residual of the conformal invariance of the (free) wave equation in $1+1$ dimensions, namely $\phi_{t t}-\phi_{x x}=0$, which is Bäcklund-transformable into the the conformal invariant Liouville equation $\phi_{t t}-\phi_{x x}=e^{\phi}$ (see e.g. Olive [13]).

5. The sine-Gordon theory can be also seen as the non-lineal sigma model on the sphere $S^{2}$ [14], [7], and therefore it is the simplest of the $\sigma$-models in $1+1$ which are exactly integrable, reflecting perhaps the fact that the naive $0+1 " \sigma$-model", the free motion on the sphere $S^{n}$, is superintegrable 15 .

All this enhances the importance of the sine-Gordon system as a toy model for some desirable properties of realistic theories, such as duality, bosonization, supersymmetry and softly-broken conformal invariance 13 .

In this paper we focus our attention in the original motivation for the appearance of the sine-Gordon equation, namely (1) in light cone (characteristic) coordinates is precisely the equation which describes the classical surfaces of constant negative curvature (Enneper, ca. 1880). Because the compact representants of these surfaces are topologically classified by the genus $g>1$, and because also the manifold of solution of equation(1) falls into classes labelled by the topological charge

$$
q(\phi)=n(\phi)=(1 / 2 \pi) \int \phi_{x} d x=(1 / 2 \pi)\{\phi(+\infty, t)-\phi(-\infty, t)\},
$$


it is natural to relate the two topological invariants. The resulting relation is explained in section 3 including the concordance of the moduli space of this surfaces under analytic transformations with the initial positions and velocities of the $n$-soliton configuration. But first in section 2 we elaborate a bit on the geometry of the equation and its solutions.

\section{Surfaces of negative curvature}

A surface $\Sigma$ embedded in ordinary space $R^{3}$ with gaussian curvature $K<0$ everywhere has two asymptotic directions in each point, separating the regions of positive and negative normal curvature $\kappa$ ( see e.g. Eisenhart [3]). Taking coordinates $u, v$ parametrized by the arc-length, along these directions, the metric becomes

$$
d s^{2}=d u^{2}+2 F(u, v) d u d v+d v^{2}
$$

where $F$ is the cosine of the angle $\phi$ of parametric lines,

$$
F(u, v)=\cos \phi(u, v) ;
$$

all information on the surface is encoded in the function $F$. It is easy to prove that these coordinates can be taken throughout the surface; this is called a "Chebichev net" on the surface 16.

The gaussian curvature is easily calculated,

$$
K=\frac{1}{1-F^{2}}\left(F_{u v}+\frac{F F_{u} F_{v}}{1-F^{2}}\right)
$$

or in terms of the $\phi$ angle

$$
\phi_{u v}=-K \sin \phi
$$

where $K$ is the gaussian curvature. If $K$ is a negative constant (e.g. $-1 / a^{2}$, say), this is of course the sine-Gordon equation (11) in light cone (or characteristic) coordinates, for $a=1$,

$$
u=\frac{t+x}{2} \quad v=\frac{t-x}{2} .
$$

For this reason, equation (8) was considered by Bianchi "l'equazione fondamentale di tutta la teoria delle superficie pseudosferique" (quoted by Coleman [6]).

On the other hand the compact oriented surfaces of negative curvature, which are Riemann surfaces, are perfectly well known and classified (see e.g. [17], 18]): there is the universal model, in the form of a simply connected space (with the topology of the plane), which is usually presented in three forms 17]:

a) The Minkowski model: the upper sheet $\mathrm{H}^{+}$of the two-sheeted hyperboloid in $R^{3}$ with the inherited metric from the ++- metric in the ambient space $R^{3}$. 
b) The Poincare disc $\Delta$, which is a stereographic projection of the former from the vertex of the lower hyperboloid

$$
\Delta=\{z \in C|| z \mid<1\} \quad d s^{2}=\frac{4\left(d x^{2}+d y^{2}\right)}{\left(1-|z|^{2}\right)^{2}} .
$$

c) The upper half plane $U$ (Klein model)

$$
U=\{z \in C \mid \operatorname{Im} z>0\} \quad d s^{2}=\frac{d x^{2}+d y^{2}}{y^{2}}
$$

$H^{+}=\Delta=U$ are connected simply connected Riemann surfaces of constant negative curvature $(-1$ by the given metric). For a detailed description of these surfaces in relation to chaotic motion see 21].

Any other Riemann surface of the conformal class $K<0$ is obtained by quotienting by a subgroup $G$ of the modular group $M$, which is a discrete automorphism group,

$$
\Sigma=U / G, \quad G \subset M=P S L(2, Z) .
$$

In fact, there are three types of these surfaces:

1. The simply connected case, say $\Sigma=\Delta$ or $U$, with the topology of the plane $C=R^{2}$; here $G=\{e\}$.

2. Those $\Sigma$ with $G=Z=\Pi_{1}(\Sigma)$, topology of the cylinder and conformally equivalent to [18]

$$
\Delta^{*}=\Delta-\{0\} \quad \text { or } \quad \Delta_{r}=\{z \in C|0<r<| z \mid<1\}
$$

3. All the other surfaces have a non-abelian, fundamental group $\Pi_{1}$, are compact and topologically homeomorphic to a sphere with $g$ handles (or holes), where $g>1$ ( $g=1$ correspond to the torus $T^{2}$, which is of the conformal class flat). They can be represented as union of tori $T=T^{2}$

$$
\Sigma_{g}=T \# T \# \cdots \# T \quad(g \text { times, } g>1)
$$

where \# means the connected sum, obtained by removing a little open disc in each torus and soldering two of them by the boundary circle 19.

4. There remain only non-orientable surfaces; the compact ones are also classified by the genus $g$, and can be obtained by the connected sum of projective planes $R P^{2}=S^{2} / Z_{2}$ (antipodal map),

$$
\Sigma_{g}^{\prime}=R P^{2} \# R P^{2} \# \cdots \# R P^{2} \quad(g \text { times, } g>1) .
$$

The case $g=1$ is the Klein bottle, of $K=0$ class. 
The homology of these surfaces is easily computed, and it is [16]

$$
\begin{aligned}
\chi\left(\Sigma_{g}\right) & =b_{0}-b_{1}+b_{2}=1-2 g+1=2(1-g) \\
\chi\left(\Sigma_{g}^{\prime}\right) & =1-g+0=1-g \\
H_{1}\left(\Sigma_{g}^{\prime}\right) & =Z^{g}+Z_{2}
\end{aligned}
$$

In all these surfaces we can chose a constant curvature metric, by Riemann uniformization theorem [18]. However, as such they cannot be embedded in $R^{3}$ with the induced metric from +++ (Hilbert theorem, 16 ).

We shall need the area of the compact oriented surfaces which might be computed from the volume element

$$
d A=\sqrt{E G-F^{2}} d u d v=|\sin \phi| d u d v
$$

The area and the Euler number are connected through the fundamental GaussBonnet formula [16]

$$
\chi=\frac{1}{2 \pi} \int K d A .
$$

This will be the key to identify the particular Riemann surfaces.

\section{Genus from soliton configurations}

The solution of the sine-Gordon equation (11) or (8) are classified by the topo-

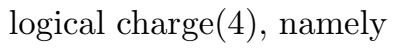

a) $q=0$; the vacuum sector. It contains the vacuum solution $\phi=2 \pi k$, the soliton -antisoliton scattering configurations, the soliton-antisoliton bound states (breather mode) and combinations thereof.

b) $q= \pm 1$; it contains one soliton (resp. antisoliton) of equation (3), traslated and/or boosted, plus any solution of a) above.

c) q arbitrary integer number; this is the multisoliton configuration; for example $q=+2$ will contain two solitons plus any solution of a) above; etc.

Which negative curvature surfaces do these configurations belong to? Let us start with the $q=2$ two soliton state. The connection with the genus of the potential surface will be made through the Gauss-Bonnet theorem: we can perform an area integration, applying to (17) and (18) the equation (8) $\sin \phi=\partial_{u v} \phi:$

$$
\int K d A=(-1) \int|\sin \phi| d u d v=(-1) \int \partial_{u v} \phi d u d v=(-1) \phi \text { (boundaries) }
$$


This is called "Hazzidaki's formula [16]. Now for the two-soliton configuration it turns out that the algebraic sum of the boundary values are just the jump in the $x= \pm \infty$ at $t=0$ minus the jump in $t= \pm \infty$ at $x=0$, due to the relation between $u, v$ and $x, t$ (9):

$$
\begin{aligned}
\text { Area }= & \phi(x=+\infty, t=0)-\phi(x=-\infty, t=0)- \\
& \{\phi(x=0, t=+\infty)-\phi(x=0, t=-\infty)\}
\end{aligned}
$$

which is just $4 \pi$ for the two-soliton state (here there is not jump in $t$ as $\phi(x=$ $0, t)=$ const.,by symmetry); the explicit formula for the two-soliton solution in $x, t$ coordinates is [1], 迎

$$
\phi(x, t)=4 \arctan \frac{-\cosh \gamma v t}{v \sinh \gamma x}
$$

where now $v$ is the relative velocity and $\gamma^{2}=\left(1-v^{2}\right)^{-1}$.

Therefore we are describing a $g=2$ surface, according the integral formula

$$
\chi=\frac{1}{2 \pi} \int K d A=2(1-g)=(-1) \frac{4 \pi}{2 \pi} \quad g=2 .
$$

The generalization to $n=2 \nu$ even number of solitons is immediate, because again there is no jump in $t$ while the jump in $x$ is given by $2 \pi n$

$$
2(1-g)=-\frac{(2 \pi)(2 \nu)}{2 \pi} \Longrightarrow g=\nu+1 \quad(\nu>0)
$$

and it describes the compact oriented surface of genus $g$. The span in $\phi$, namely $4 \pi \nu$, reflects the "holes" of the surface. This is a satisfactory result. Of course, the integration can be performed analytically also. The configuration with $2 n$ antisolitons will presumably describe the same surface with changed orientation.

The correspondence goes along also with the integration parameters; namely we can choose three integration constants for each soliton, the center, origin of time and velocity (the three parameters of the $1+1$ Poincare group). But the moduli space of the surface of genus $g$ is known to be the Teichmüller space [17], 18 of complex dimension 20

$$
B_{g}=\operatorname{dim} \operatorname{Teich}\left(\Sigma_{g}\right)=3(g-1)=\frac{1}{2} \times 3 \times(2 \nu)=\frac{1}{2}(\# \text { real parameters })
$$

because $g=\nu-1$. So this is again in agreement.

We do not have a satisfactory answer for the odd-soliton case, for which the integration is ill-defined. If we maintain (22) for any soliton number, i.e.

$$
-\chi=n \Longrightarrow g=n+1 \text { for unoriented surfaces, } \quad \chi=1-g
$$

and we conjecture that this is true; in this case the soliton will describe the "unoriented" pretzel, $g=2$. This goes on with the fact that the soliton will be 
a fermion, and fermions are odd under full rotations. Again the concordance goes also with the moduli space, for which the freedom is now in real dimension 117

$$
B_{g}=3(g-1)=\text { \#param. of the } \mathrm{n} \text {-sol. config. }
$$

There are still other surfaces (Cfr. section 2) ; we conjecture also that the breather mode, i.e. a non trivial solution with $\mathrm{q}=0$, will correspond to the non-compact case, e.g. to the simply connected model $\Delta$ or $\mathrm{U}$.

\section{Acknowledgements}

Discussions with M. Asorey and F. Falceto (Zaragoza) and M. Santander (Valladolid) were useful. This work was supported in part by research grants AEN961670 (CSIC) and ERBCHRX-CT92-0035.

\section{References}

[1] J. Rubinstein, Sine-Gordon equation, J. Mat. Phys. 11 (1970) 258-266. Reprinted in [2].

[2] C. Rebbi and G. Soliani eds., Solitons and Particles, (World Scientific, Singapore,1984).

[3] L. Eisenhart, Differential Geometry of Curves and Surfaces (1909), (Dover, N.Y., 1960), p.284.

[4] R. Dodd, J.C. Eilbeck, J. Gibbon and H.C. Morris, Solitons and Nonlinear Wave Equations, (Academic Press,N.Y.,1983), pp. 14ff.

[5] M.J. Ablowitz, D.J. Kaup, A.C. Newell and H. Segur, Method for solving the Sine-Gordon equation, Phys. Rev. Lett. 30 (1973), 1095-1097. Reprinted in [2]

[6] S. Coleman, in Proc. Erice Summer School,1975. Reprinted in Aspects of Symmetry, (Cambridge U.P.,1985),p.223.

[7] A.B. Zamalodchikov and A.B. Zamalodchikov, Factoriced S-matrix in Two Dimensions as the Exact Solutions of Certain Relativistic Quantum Field Theory Models, Ann. Phys. 120 (1979), 253-291. Reprinted in [2].

[8] R.F. Dashen, B. Hasslacher and A. Neveu, Particle spectrum in model field theories from semiclassical functional integral techniques, Phys. Rev. D11 (1979), 3120-3130.

[9] S. Coleman, Quantum sine-Gordon equation as the massive Thirring model, Phys Rev. D11 (1975), 2088-2097. Reprinted in [2]. 
[10] T.H.R. Skirme, A non-linear theory of strong interactions, Proc. Roy. Soc. A247 (1958),260-278. Particle states of a quantized field, Proc. Roy. Soc. A252 (1961), 237-245.

[11] S. Mandelstam, Soliton operators for the quantized sine-Gordon equation, Phys. Rev. D11 (1975), 3026-3028.

[12] P. Di Vecchia and S. Ferrara, Classical solutions in two-dimensional supersymmetric field theories, Nucl. Phys. B130 (1977), 93-104. J. Hruby, On the supersymmetric sine-Gordon model and a two dimensional bag, Nucl. Phys. B131 (1977), 275-284.

[13] D.I. Olive, Exact electromagnetic duality, Nucl. Phys. B (Proc. Supl.) 45A (1996), 88-102.

[14] K. Pohlmeyer, Integrable hamiltonian systems and interactions through quadratic constraints, Comm. Math. Phys.46 (1976), 207-221.

[15] L. J. Boya, Free motion on the sphere, Int J. Mod. Phys. A9 (1994), 54555468.

[16] M. Spivak, Differential Geometry, Vol.III, Ch.5, (Publish or Perish, Berkeley(CA), 1979).

[17] M. Berger and B. Gostiaux, Differential Geometry, Grad. Texts in Math., (Springer,Berlin,1988),Ch.11.

[18] H.M. Farkas and I. Kra, Riemann Surfaces, (Springer,Berlin,1980),Ch.1.

[19] J.R. Munkres, Elements of Algebraic Topology, (Addison-Wesley,Menlo Park,CA,1984),p.38.

[20] An intuitive derivation of the moduli space formula for Riemann surfaces is given in the book by Witten-Green-Schwartz, Superstrings, (Cambridge U.P.,1989),Vol.I,p.159.

[21] N.L. Balasz and A. Voros, Chaos in the pseudoesphere, Phys. Rep. 143 (1986),109-420. 